\title{
Evaluación del aprendizaje de los estudiantes de las carreras de medicina y odontología dentro de un modelo de atención interdisciplinario a nivel hospitalario, Gentro Hospitalario Pereira Rossell, Montevideo Uruguay
}

\author{
Evaluation of the learning process of medicine and dentistry students \\ within an assistential interdisciplinary teaching model at a hospital level, \\ Hospital Center Pereira Rossell, Montevideo Uruguay
}

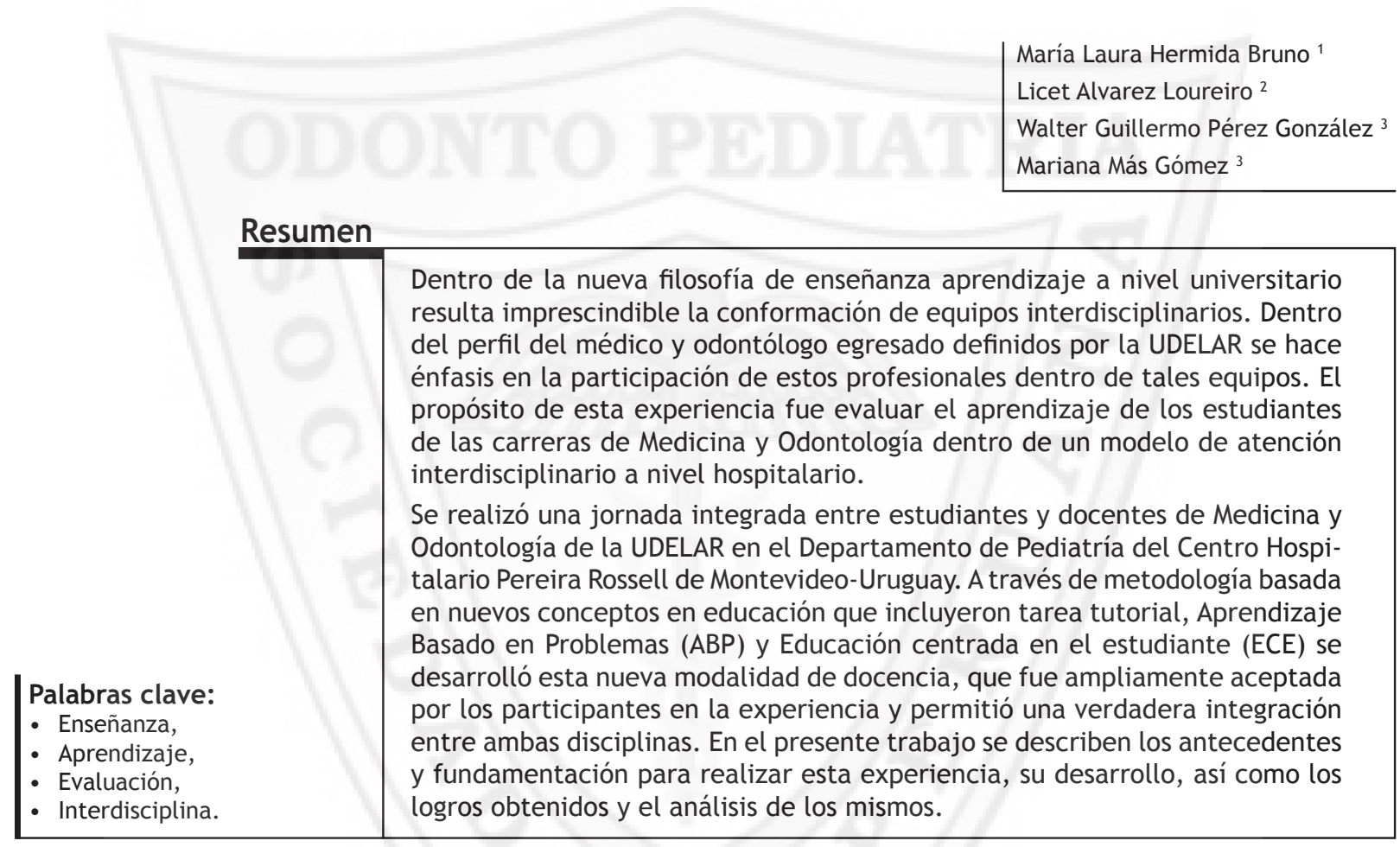

Abstract

Keywords:

- Teaching,

- Learning,

- Evaluation,

- Interdiscipline.
In the health field it is important that graduate students have a profile that allows them to participate in interdisciplinary teams. The aim of this study was to evaluate the learning process in Medicine and Dentistry students within an assistential interdisciplinary teaching model at a hospital level.

A study was developed with the participation of Dentistry and Medicine proffessors and students, at the Pediatric Department in the Pereira Rossell Hospital Center. It was done a pre-test, a general presentation by the teacher team involved in this project, clinic examination of some patients previously selected, case report and post test. Diagnostic, formative and summative concepts of evaluation and self evaluation were taken into consideration. For evaluation it was used cuantitative and qualitative methodology. The results indicated that this experience was widely accepted for all participants. (OdontolPediatr2009; 8(1):4-8)

Trabajo realizado en el Centro Hospitalario Pereira Rossell en Montevideo, Uruguay.

1. Docente Programa Docencia Servicio Investigación, Area del Niño. Facultad de Odontología, Universidad de la República (Montevideo, Uruguay). Especialista en Docencia Universitaria. Universidad El Bosque (Bogotá, Colombia).

2. Coordinadora Programa Docencia Servicio Investigación, Area del Niño. Docente Odontopediatría. Facultad de Odontología, Universidad de la República (Montevideo, Uruguay).

3. Docente de Clínica Pediátrica- Facultad de Medicina, Universidad de la República (Montevideo, Uruguay). 


\section{Introducción}

Dentro de la nueva filosofía de enseñanza aprendizaje a nivel universitario resulta imprescindible la conformación de equipos interdisciplinarios tanto para el desarrollo de programas conjuntos entre las distintas disciplinas involucradas en la resolución de problemas como para la evaluación del aprendizaje de los estudiantes y de los propios programas. En las carreras de la salud, dentro del perfil del egresado se enfatiza la importancia de la capacitación para participar en equipos interdisciplinarios, definidos como "grupos de personas con habilidades diferentes, que dependen unas de otras para funcionar eficientemente y para lograr metas y objetivos comunes". ${ }^{1}$ El plan de estudios 1993 de la Facultad de Odontología de la Universidad de la República (UDELAR) define la formación de un odontólogo con una perspectiva clínico-epidemiológicosocial que enfatiza la prevención, capaz de interactuar en equipos interdisciplinarios, interprofesionales, asegurando una cobertura de salud universal, integral, accesible, contínua y oportuna, priorizando una visión científica de su práctica y con una capacidad de organizar, planear y administrar servicios de salud ${ }^{2}$. Por otra parte, el perfil del médico egresado de la Universidad de la República define "un médico responsable, con sólida formación científica básico-clínica, capaz de realizar diagnósticos correctos, tomar decisiones clínicas precisas, capaz de comunicarse en su misión de prevenir, curar y derivar adecuadamente; profundamente humanista, crítico, preparado para investigar, educar y educarse, dispuesto siempre a aprender, proporcionándole durante su preparación las herramientas metodológicas imprescindibles a tal fin; comprometido con la ética, preparado conscientemente para trabajar en un sistema que priorice la atención primaria de la salud, adiestrado para interactuar armónicamente con los otros profesionales de la salud; que es, en definitiva, lo que demanda nuestra sociedad". ${ }^{3}$

Modernizar y diversificar la estructura académica de la Universidad de la República (UDELAR) es una de las tareas mayores de la Reforma Universitaria. Posibilitar su realización integral es uno de los principales motivos para impulsar la modificación de la Ley Orgánica de la UDELAR.

La tarea requiere conjugar cambios legales y acciones concretas. Un paso, entre otros en esa dirección, lo constituye la construcción del Espacio Interdisciplinario (EI). ${ }^{4,5}$

Dentro de los objetivos estratégicos más importantes del Plenario de la UDELAR (PLEDUR) podemos citar: Impulsar la creación científica, tecnológica y artística estimulando su calidad, promover la utilidad social del conocimiento contribuyendo a la solución de los problemas que hacen a la mejora de la calidad de vida de la población e impulsar procesos de modernización en la gestión capaces de sustentar eficientemente las transformaciones de la UDELAR. Este último orientado a mejorar la gestión académica institucional y técnico administrativa. ${ }^{6}$

Para el desarrollo de los procesos de flexibilización curricular planteados por la Universidad de la República resultan esenciales las diferentes modalidades de tutorías. Este es uno de los pilares de la Educación Centrada en el Estudiante (ECE), que concibe la formación del estudiante como gestor y consciente de su propia construcción y transformación, que permite su crecimiento y el conocimiento de sus capacidades mentales, afectivas, volitivas y psicomotoras y el avance tanto de su desarrollo personal como de la adquisición de conocimientos. Todo esto hace indispensable la autoevaluación, porque es el mismo educando quien necesita identificar sus aciertos y sustentarlos, sus errores para corregirlos, de modo que tome conciencia de su desarrollo y se forme como persona autónoma, capaz de autoeducarse permanentemente. $7,8,9$

La tarea tutorial cumple un rol central en el método de Aprendizaje Basado en Problemas (ABP), en el cual se busca la interacción del grupo y el logro del consenso del mismo para la explicación de los fenómenos y las soluciones propuestas. ${ }^{10,11}$

La evaluación del aprendizaje puede definirse como la identificación y verificación de los conocimientos, los objetivos, las habilidades, no con el fin de dar una nota sino de observar y analizar cómo avanzan los procesos de aprendizaje y formación implementados. ${ }^{12}$

Los estudiantes de las carreras de Medicina y Odontología durante su último año curricular desarrollan actividades en ámbitos hospitalarios comunes. Los estudiantes de Medicina cursan el Ciclo Materno Infantil en el Centro Hospitalario Pereira Rossell (Departamento de Pediatría y Clínicas Gineco obstétricas) y en policlínicas comunitarias del subsector público (ASEE,IMM, BPS). Del mismo modo, los estudiantes del último año de la carrera de Odontología pueden cursar sus pasantías de atención odontológica en Servicios y Comunidad II en el mismo Centro Hospitalario.

Sin embargo, a pesar de lo expresado anteriormente en el sentido de que ambas Facultades dentro de su plan de estudios contemplan la necesidad de formar a los estudiantes dentro de modelos de atención interdisciplinarios, esto no se ve reflejado en el desarrollo de los programas académicos y las metodologías utilizadas en las pasantías estudiantiles. Por otra parte, existen reportes acerca de la falta de conocimiento de los docentes sobre los documentos referidos a ciertos aspectos relacionados con la formación de los estudiantes tales 
como los fines de la Universidad, el perfil del egresado y los objetivos de aprendizaje en determinadas disciplinas. ${ }^{13}$

Consideramos que es necesario diseñar estrategias e instrumentos que permitan desarrollar programas conjuntos y evaluar la utilización de este modelo de atención interdisciplinario, que a su vez se basa en los conceptos antes mencionados, así como evaluar el aprendizaje de los estudiantes dentro de dicho modelo.

Por lo tanto, el propósito de esta experiencia fue evaluar el aprendizaje de los estudiantes de las carreras de Medicina y Odontología dentro de un modelo de atención interdisciplinario a nivel hospitalario.

\section{Material y métodos}

Como resultado de la iniciativa de docentes de la Facultad de Odontología y Medicina, se conformó un grupo para trabajar en la evaluación del aprendizaje en el espacio interdisciplinario. Estaba integrado con dos docentes de la Cátedra de Pediatría B de la Facultad de Medicina y dos integrantes del área pediátrica del Programa Docencia servicio investigación, de la Facultad de Odontología de la UDELAR. Una vez determinados los objetivos disciplinares su cometido fue puntualizar los problemas concretos de interés común para buscar la integración y producción de conocimiento. El punto de partida sería la situación problema y no las disciplinas individuales.

Para la experiencia interdisciplinaria se utilizó la modalidad de jorna$\mathrm{da}$, trabajando en forma conjunta estudiantes del último año de las mencionadas carreras, en el Departamento de Pediatría del Centro Hospitalario Pereira Rossell de Montevideo, Uruguay, en dos opor- tunidades y con grupos diferentes. La integración se realizó en una actividad teórico-práctica participativa (taller) donde se compartieron los objetivos de aprendizaje desde sus propias perspectivas.

En la primera jornada participaron de la experiencia 15 estudiantes de medicina que estaban cursando el CMI en el marco del curso de tratamiento y 15 estudiantes de la Facultad de Odontología que comenzaban su pasantía hospitalaria dentro del Programa "Atención Odontológica en Servicios y Comunidad II" ${ }^{14}$. En la segunda jornada el número de estudiantes para ambos grupos fue 12 .

Al comienzo del taller se entregó a cada participante un test de conocimientos previos, compuesto de 6 preguntas, diferenciado para los estudiantes de las dos carreras. Los estudiantes se registraron con un seudónimo porque la finalidad del test no era la evaluación sumativa sino diagnóstica, buscando identificar los conocimientos iniciales de los temas previamente puntualizados por los docentes. A continuación los estudiantes se presentaron individualmente y como grupo.

Los docentes realizaron una ponencia integrada de 40 minutos, de estilo incitativo, sobre aspectos básicos que incluían variantes normales de desarrollo, semiología y patologías más prevalentes en niños y adolescentes, cuyos contenidos reflejaban las conceptualizaciones esperadas en el pre-test.

Se conformaron equipos de trabajo integrados por estudiantes de ambas carreras asignándole a cada uno un paciente, que previamente había sido seleccionado y observado por los docentes. Como instrumento de debate se les entregaba la historia clínica del paciente y se les daba una guía orientadora con preguntas a contestar sobre el caso. Disponían de 60 minutos para la evaluación integral del paciente e intercambio de información. Debían realizar la semiología oral y general, elaborar un diagnóstico y definir estrategias de tratamiento y seguimiento. Los docentes estaban presentes para colaborar en la instancia de intercambio y como observadores de la actividad.

Luego todos los grupos se reunieron en plenario. Un delegado de cada grupo contestó las guías de trabajo elaboradas en conjunto. El resto del grupo realizó los aportes que consideró convenientes. Los docentes moderaron el plenario y colaboraron en las conclusiones.

Antes de retirarse realizaron nuevamente el test inicial (post-test) permitiéndoles comparar los resultados con el pre-test y analizar los posibles logros en el área del conocimiento. El equipo docente al finalizar realizó la devolución de la jornada, aclarando aquellas dificultades que pudieron surgir y puntualizando aciertos y dificultades de la experiencia misma. Se analizaron las respuestas del test.

Luego de la primera Jornada, el equipo docente realizó una evaluación de todos los aspectos de la misma, tanto operativos como los aportados por los estudiantes, y esto permitió realizar ajustes y cambios para el desarrollo de la segunda Jornada. Posterior a ésta nuevamente se realizó la evaluación, para permitir experiencias futuras en esta disciplina $\mathrm{y} / \mathrm{o}$ en otras de las respectivas carreras.

Para la evaluación se utilizó metodología cuantitativa y cualitativa. Para la evaluación cuantitativa del parámetro "conocimientos" se utilizaron promedios y porcentajes, mientras que para la evaluación cualitativa que analizó las habilidades, destrezas y actitudes se utilizó un diseño de teoría fundamentada e investigación-acción. Se tuvieron en 
cuenta los criterios de credibilidad, transferibilidad y confirmabilidad con el fin de aumentar la calidad y objetividad de los datos y el análisis. La evaluación cualitativa se realizó a través de análisis cuasi estadístico de tipo descriptivo y análisis mediante plantilla. ${ }^{15}$

El equipo docente realizó un informe de ambas experiencias y lo elevó a sus respectivos centros de estudio.

\section{Resultados}

Los resultados fueron los siguientes:

En la primera jornada el grupo de estudiantes de medicina obtuvo un pre test: $53 \%$ respuestas correctas en el pre-test mientras que en el post test el resultado fue de $93 \%$. En la misma Jornada el resultado del pre test de los estudiantes de Odontología fue de $71 \%$ de respuestas correctas, mientras que en el post test el mismo grupo logró el $100 \%$. (Tabla No 1).

En la segunda jornada el grupo de estudiantes de medicina alcanzó el $36 \%$ respuestas correctas en el pre test y luego logró el $87 \%$ de respuestas correctas en el post test. El grupo de odontología obtuvo un 33\% de respuestas correctas en el pre test y $68 \%$ en el post test. (Tabla No 2 ).

Se observó un cambio de actitud notorio desde el inicio a la finalización de la Jornada por parte de los estudiantes participantes en la misma. Los docentes tuvieron únicamente un papel facilitador, siendo los estudiantes los verdaderos protagonistas en el proceso. Se manifestó una percepción positiva hacia la odontología por parte de los estudiantes de Medicina, que hicieron notar una sentida necesidad en recibir mayores conocimientos en esta área, así como de los estudiantes de odontología con respecto a su participación en equipos multidisciplinarios.

Se percibió también una actitud de intercambio de información en las respectivas áreas semiológicas.

\begin{tabular}{|ccc|}
\hline \multicolumn{3}{c|}{ TABLA 1 . PRIMERA JORNADA } \\
\hline Pre test \% & Post test \% \\
\hline Medicina & 53 & 93 \\
\hline Odontología & 71 & 100
\end{tabular}

Porcentaje correspondiente a la prueba escrita al principio y final de la Primera Jornada para los estudiantes de Medicina y Odontología.

\section{Discusión}

No conocemos antecedentes en nuestro país de experiencias similares a ésta en lo relativo a la interacción de los estudiantes de diferentes carreras dentro de un modelo interdisciplinario. Podemos analizarla con respecto a los diferentes parámetros estudiados utilizando los conceptos de APB y ECE y en este sentido podemos afirmar que los resultados obtenidos concuerdan con lo reportado por diferentes autores en cuanto al logro de conocimientos, habilidades, destrezas y actitudes. ${ }^{7,16}$

Se despertó en los estudiantes la necesidad de mayor intercambio entre ambas Facultades, aunque esto también se vio reflejado en la evaluación que realizaron los docentes. La integración se evidenció tanto a nivel estudiantil como docente, siendo la base para otros proyectos en curso.

Consideramos que esta metodología se ajusta a las necesidades de formación de un recurso humano que responda a los requerimientos actuales de la sociedad. El cambio teórico que se operó en la conceptualización pedagógica y en los en- foques metodológicos en la década de los 80 y principios de los 90 a nivel mundial, obliga a los centros educativos universitarios a presentar un giro en la concepción de la pedagogía y la didáctica. ${ }^{17} \mathrm{Si}$ bien está planteada esta necesidad en los planes de estudio de ambas facultades, es necesario la formación del recurso humano dentro de esta concepción para responder a este requerimiento.

Cabe destacar que más allá de los resultados numéricos de los tests realizados por los diferentes grupos de estudiantes, en todos se evidencia un nivel más alto en el post test que en el pre test, lo que muestra la adquisición de conocimientos por parte de todos los grupos y la efectividad de esta modalidad de trabajo. Por otra parte, en el aspecto de actitudes y en la devolución de cada experiencia, dentro del análisis cualitativo, tanto el grupo de estudiantes como el de docentes calificó la experiencia como altamente enriquecedora. La metodología cualitativa utilizada permitió analizar la visión y percepción de los estudiantes y docentes con respecto a la propuesta planteada. 


\section{Conclusiones}

1. Se observó una evaluación positiva en todos los parámetros estudiados: conocimientos, habilidades, destrezas y actitudes.
2. La metodología utilizada fue ampliamente aceptada por todos los participantes en las Jornadas.

\section{Referencias}

1. Facultad de Medicina, U de Bs As, Fundación W K Kellogg. EDISA. Interdisciplina en los equipos de salud. Módulo No 7, 1997.

2. Plan de estudios, Facultad de Odontología, UDELAR, 1993.

3. Perfil del egresado de Facultad de Medicina, UDELAR, aprobado por el Claustro de Facultad de Medicina, 1995.

4. Arocena R. Espacio interdisciplinario en la transformación de la estructura académica. Hacia la reforma universitaria. UDELAR, Agosto 2007.

5. Consejo Directivo Central de la Universidad de la República. Ley de Educación: plan estratégico Nacional en Ciencia, Tecnología e Innovación. Marzo- Agosto 2007.

6. Rectorado UDELAR. Plan estratégico de desarrollo de la Universidad de la República 2005-2009. Setiembre de 2005.

7. Hermida L. Propuesta de estrategias para la evaluación del aprendizaje en la Facultad de Odontología de la Universidad El Bosque, Bogotá, Colombia. Tesis de Grado. Especialización en Docencia Universitaria, 1998.

8. lafrancesco G. Nueve problemas de cara a la renovación educativa: Alternativas de solución. Biblioteca básica del educador. CAD, 1997.

9. López J N. Retos para la construcción curricular. Col Mesa Redonda, 1997.
10. Careaga A, Gómez A, Musetti G, Scocozza M. La acción tutorial. Universidad de la República, Facultad de Medicina, Departamento de Educación Médica, Julio 2006.

11. Wang G, Tai B, Huang C, Bian Z, Shang Z. Establishing a multidisciplinary PBL curriculum in the School of Stomatology at Wuhan University. J Dent Educ, Vol 72, Number 5, 2008.

12. AFACIMERA. Conceptos básicos de Evaluación Educacional. Módulo No 1, 2002.

13. Bernadá M, Dall'Orso P, Zabala C. Encuesta a docentes del ciclo materno infantil: conocimiento de documentos universitarios. Rev Med Urug 2006; 22: 203-209.

14. Ringel R, Blanco S, Cánepa C, Guelfi C y Visconti M. Enseñanza Aprendizaje en servicios de salud y comunidad. Fac. de odontología Universidad de la República. Programa docenciaservicio-investigación- Comisión sectorial de Investigación científica 1999.

15. Pineda E; Alvarado E. Metodología de la investigación. OPS, $3^{\text {a }}$ Edición, 2008.

16. Schönwetter D, Lavigne S, Mazurat R, Nazarko O. Student's perceptions of effective classroom and clinical teaching in Dental Education. J Dent Educ, Vol 70, Number 6, 2006.

17. A.C.F.O., División de Educación. Plan de desarrollo. Santafé de Bogotá, 1998. 